\title{
Inhibition of MDR1 in Mammary Cell Carcinoma Reverses Multidrug Resistance by SOCS1
}

\author{
Debasish Pradhan*, Gitanjali Tripathy, Rakesh Kumar Pradhan, Shaktiprasad Pradhan, Soumyashree Rupambika Moharana \\ University Department of Pharmaceutical Sciences, Utkal University, Vani Vihar, Bhubaneswar, Odisha, INDIA.
}

\begin{abstract}
Introduction: Suppressors of cytokine signalling (SOCS1), a newly indentified antiapoptotic molecule is a downstream effector of the receptor tyrosine kinase-Ras signalling pathway. Current study has uncovered that SOCS1 may have wide and imperative capacities, particularly because of its close correlation with malignant tumors. Methods: To investigate the impact of SOCS1 on MDR, we analyzed the expression of P-gp and SOCS1 by immunohistochemistry and found there was positive correlation between them. At that point we effectively interfered with RNA translation by the contamination of siRNA of SOCS1 into MCF7/ ADM breast cancer cell lines through a lentivirus, and the expression of the target gene was significantly inhibited. Results: After RNAi the drug resistance was reduced altogether and the expression of MDR1 mRNA and P-gp in MCF7/ADM cell lines demonstrated a significant decrease. Likewise the expression of P53 protein increased in a statistically significant manner $(p \leq 0.01)$ after RNAi exposure. Moreover, flow cytometry
\end{abstract}

analysis uncovers that cell cycle and anti-apoptotic enhancing capacity of cells changed after RNAi treatment. Conclusion: These outcomes proposed SOCS1 may take part in breast cancer MDR by managing MDR1 and P53 expression, changing cell cycle and enhancing the anti-apoptotic ability of cells.

Key words: Breast cancer, Multidrug resistance, SOCS1 gene, MDR1gene, RNA interference.

Correspondence:

Dr. Debasish Pradhan, University Department of Pharmaceutical Sciences, Utkal University, Vani Vihar, Bhubaneswar, Odisha, INDIA.

Phone no: +919861137772

E-mail: deba_udps@yahoo.co.in

DOI: $10.5530 /$ pj.2016.2.2

\section{INTRODUCTION}

Breast cancer is one of the common cancers everywhere throughout the World. ${ }^{1}$ There are around 1,250,000 ladies suffering breast cancer around the globe, 550,000 of which die of this disease every year. On the planet, rate of breast cancer tends to increase year by year. ${ }^{2}$ Breast cancer often has a poor survival rate because of late clinical presentation and rapid progression. Although recent improvements in early detection, surgical techniques and chemoradiotherapy have successfully advanced progressions in its treatment, its overall survival rate is still low. ${ }^{3}$ Because of the significance of chemotherapy in treating breast cancer, the improvement of multidrug resistance (MDR) turns into a serious obstacle to successful chemotherapy. ${ }^{4}$ In spite of the fact that the molecular mechanism(s) of the MDR have not yet been elucidated in breast cancer cells, a few studies have reported that the mechanisms of MDR were close connected with the overexpression of P-glycoprotein (P-gp) encoded by MDR1 gene in tumor cells. ${ }^{5-7}$ Hence, the inhibition of P-gp expression in tumor cells could be a standout amongst the best approaches to reverse MDR and make tumor cells resensitize to chemotherapy. ${ }^{8}$

Suppressors of cytokine signalling 1 (SOCS1) is a novel antiapoptotic molecule. Compared with the Bcl-2 family, caspase family and signal transduction molecule, it is a distinct apoptosis regulatory molecule and it influences the RAS signaling pathway as a mediator. ${ }^{9}$ Above all, it plays a vitally imperative part in malignant phenotypes of a few cancers. Some past studies reported that SOCS1 could influence the cell proliferation and cell cycle progression and it might be included in regulating MDR in a few cancers. ${ }^{10-16}$ However, until now no article has reported whether SOCS1 is connected with the mechanism of MDR in breast cancer cells or not. These discoveries lead to the speculation that SOCS1 may impact the MDR of breast cancer cells through cell cycle regulation, apoptosis and the expression of the MDR1 gene. The point of this study was to investigate the relationship between SOCS1 and MDR of breast cancer cells. As far as anyone is concerned, this article is the first to report an assessment of SOCS1 in reversing MDR of breast cancer/breast cell growth.

\section{RESULTS AND DISCUSSION RESULTS}

Expression and Relationship of P-gp, SOCS1 and p53 Protein in Breast cancer Patient Samples and Cell Lines

To discuss the relationship of P-gp, SOCS1and p53 primarily in breast cancer, immunohistochemistry was performed on 41 breast cancer tissue samples following the manufacture's instruction. P-gp expression had a positive rate of $51.2 \%(21 / 41)$, SOCS1 of $34.1 \%$ (14/41), P53 of $41.5 \%$ (17/41), which had a significant difference comparing with the normal breast tissue around the cancer $(p<0.05)$. P-gp protein was expressed in cytoplasm and cell membrane, while SOCS1 was in the cell nucleus (Figure 1). The P-gp expression showed a positive correlation with the expression of SOCS1 $(p<0.01)$ and of P53 $(p<0.025)$ (Table 1). Immunohistochemistry tests thus implied that SOCS1 and p53 participated in multi-drug resistance in breast cancer, and that it might participate through regulation of $\mathrm{P}$-gp expression.

To investigate the differences of SOCS1 levels in various breast cancer cell lines, we analyzed by Western blot the amount of SOCS1 protein. Results showed the significant increase of SOCS1 protein level in MCF7/ ADM cells possessing MDR properties compared with MCF7 cells (Figure 2). In other words, there was the significant difference in the expression level of SOCS1 protein between MCF7 and MCF7/ADM cells.

Table1: Relationship among the expression of P-gp, SOCS1 and P53 in breast cancer tissues

\begin{tabular}{ccccccc}
\hline \multirow{2}{*}{ P-gp } & \multicolumn{3}{c}{ SOCSI } & & \multicolumn{3}{c}{ P53 } \\
\cline { 2 - 7 } & + & - & $\mathrm{P}$ & + & - & $\mathrm{P}$ \\
\hline+ & 11 & 10 & $<0.01$ & 13 & 8 & \\
- & 3 & 17 & & 4 & 16 & $<0.025$ \\
\hline
\end{tabular}




\begin{tabular}{|c|c|c|c|}
\hline \multirow{2}{*}{ Group } & \multicolumn{3}{|c|}{$\mathrm{IC}_{50}$ Value } \\
\hline & Paclitaxel & Epirubicin & Gemcitabine \\
\hline MCF7 & $0.808 \pm 0.004$ & $1.067 \pm 0.016$ & $5.859 \pm 0.551$ \\
\hline MCF7/ADM & $7.121 \pm 0.312$ & $11.206 \pm 1.789$ & $49.724 \pm 4.522$ \\
\hline MCF7/ADM + SOCSI-RNAi & $1.134 \pm 0.057^{*}$ & $4.514 \pm 0.203^{*}$ & $18.296 \pm 1.273^{*}$ \\
\hline
\end{tabular}

MCF7/ADM RNAi verse MCF7/ADM $P \leq 0.01$.

\section{Identification of Recombined Plasmid of siRNA Targeting SOCS1 and Selection of the Best Interference siRNA Sequence}

DNA sequencing was conducted on every siRNA and the sequences were proved to be the same as designed, which proved the oligonucleolide fragments were successfully inserted into the pSIH1-H1-copGFP vector. We transiently transfected recombined plasmid into 293 cells and observed cell growth condition with a fluorescent microscope. In the fluorescent visual field, we observed transfection efficiency was $90 \% 48 \mathrm{~h}$ after transfection. Among three siRNAs targeting SOCS1, SOCS1mRNA and protein expression are the lowest in SOCS1-siRNA1 which is the best interference siRNA sequence and was chosen for use in the next experiment (Figure 3).

\section{RNA Interference Test by Lentiviral-Based Vector}

We infected lentiviral expressed vector into MCF7/ADM cells (MOI=6) and obtained a stably expressed cell line after screening. The gene SOCS1 silencing efficiency is $88 \%$ as detected by Real Time-PCR while the inhibition efficiency of protein expression level is $83 \%$ by Western blot, which reported that we successfully accomplished RNA interference targeting SOCS1 through the lentivirus. Also the expression of Green Fluorescent Protein (GFP) and target gene showed no change at various clone ages, which proved the MCF7/ADM-SOCS1 RNAi cell line is of good quality (Figures 4 and 5).

\section{SOCS1 siRNA Reversing the Multidrug Resistance}

We measured the IC50 values of MCF7/ADM cells exposed to several clinical chemotherapeutics (epirubicin, paclitaxel, gemcitabine) and observed that they decreased significantly after RNAi to the same extent as MCF7 cells (Table 2). The drug resistance was reduced significantly. We concluded that RNA interference targeting SOCS1 can reverse the MDR properties of breast cancer efficiently.

\section{SOCS1 siRNA Down Regulate Expression of MDR1}

To investigate the effect of SOCS1 on MDR1 expression of breast cancer cells, MDR1mRNA and P-gp of MCF7/ADM cell were determined by Realtime PCR and Western blot, respectively, under SOCS1 gene silencing conditions. As expected, the expression of MDR1mRNA and P-gp in MCF7/ADM cell lines displayed a statistical significance before and after RNAi $(p \leq 0.01)$ exposure. These results indicated that SOCS1 may affect MDR-1 to inhibit P-gp expression in drug-resistant MCF7/ADM cells (Figure 6). We got a similar result in a Rhodamine 123 staining test (Figure 7). The fluorescence intensity of MCF7/ADM is distinctly lower than its parental cell line MCF7, which shows that the drainage ability is stronger in the drug resistant cell line, while in MCF7/ADM siRNA cell line the fluorescent intensity was enhanced remarkably, which means the drainage ability is weakened after RNAi exposure when SOCS1 is silenced.

\section{Cell Cycle Analysis}

Our flow cytometry analysis results showed that the cell cycle distribution was significantly affected by siRNA targeting SOCS1. The cell cycle profile indicates that the number of cells in G1 phase increased markedly from $(46.60 \pm 4.27) \%$ before RNAi to $(75.30 \pm 4.80) \%$ after RNAi, while those in S phase decreased from $(42.09 \pm 6.04) \%$ to $(18.81 \pm 5.18) \%$ (Figure 8 ). These results suggested that siRNA targeting SOCS1 inhibited the entry of cells into S phase, hence indicating that SOCS1 exerted a promoting effect on cell cycle progression which might partially participate in the MDR process.

\section{Apoptosis Analysis}

We postulated that the effect of SOCS1siRNA on MCF7/ADM was related to its impact on apoptosis. To test this assumption, we stably transfected SOCS1siRNA into MCF7/ADM cells. Apoptosis of cells were determined by flow cytometry analysis. Compared with the cells without the SOCS1 siRNA treatment, the apoptosis rate increased significantly from $(17.83 \pm 2.24) \%$ before RNAi to $(73.52 \pm 7.95) \%$ after RNAi (Figure 9). These results demonstrated that SOCS1 siRNA could induce apoptosis of MCF7/ADM cells.

\section{SOCS1siRNA Influenced the Expression of P53}

We detected the influence of RNA interference targeting SOCS1 on P53 by Western blot. The results indicated that the protein expression of P53 in MCF7/ADM cell lines increased after RNAi which means that SOCS1 may participate in MDR of breast cancer by regulating P53 expression (Figure 10).

\section{DISCUSSION}

As we have described, breast cancer is one malignant tumor which endangers the health of women. Many routine chemotherapies achieve poor therapeutic effects as well as bad prognosis, both of which cause huge clinical problems. It is believed that MDR is the key factor in the failure of breast cancer treatments. There are many complicated mechanisms for causing MDR. ${ }^{17-21}$ Study on MDR reversal by drugs has been performed for over 20 years, however, many problems still need to be solved. Finding and studying genes related with MDR of breast cancer as well as their mechanisms for obtaining new targets which can improve therapeutic effects has become a current hot-spot for medical research. SOCS1 is a new apoptosis-regulating molecule independent of the bcl-2 family, and caspase family. It is produced by stimulation of many cell factors, which widely exist in all kinds of normal tissues of fetus and adult, especially in hematopoietic organs like the liver and spleen of fetus as well as in tissues with higher metabolism, where expression of SOCS1 is relatively high. ${ }^{22}$ Although the physiological functions of SOCS1 have not been fully understood, however, current study has revealed that SOCS1 may have wide and important functions, especially its close correlations with malignant tumors. A primary study found that SOCS1 may protect mouse IL-3-dependent cell line Ba/F3 from etoposide and $\gamma$-rays. ${ }^{9}$ In recent years, more studies also confirmed that 


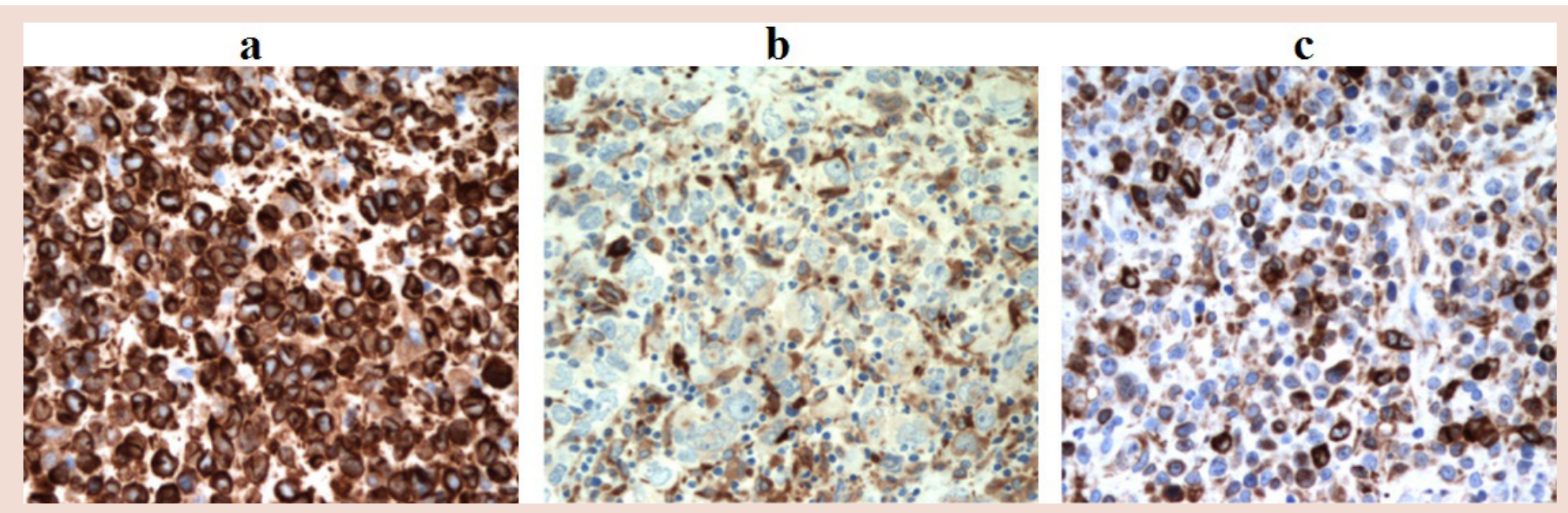

Figure 1: Immunohistochemical analysis of $\mathrm{P}-\mathrm{gp}$, SOCS1 and P53 protein expression in breast cancer tissues (41 cases): (a) expression of $\mathrm{P}-\mathrm{gp}$ (SP, $\times 400)$; (b) expression of SOCS1 (SP, $\times 400)$; (c) expression of P53 (SP, $\times 400)$.
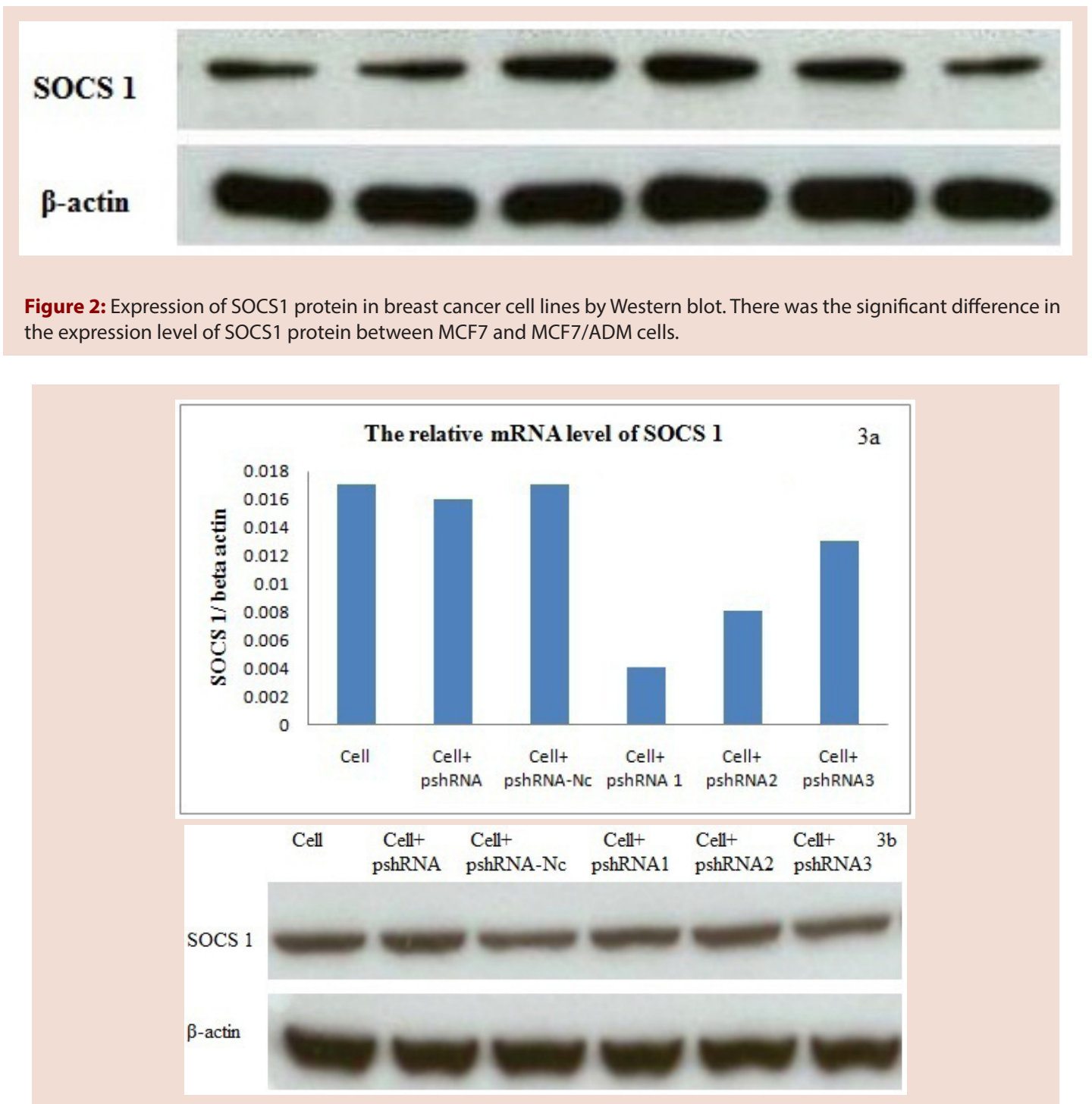

Figure 3a,b: Expression of SOCS1 mRNA and protein in each cell line after transfection. Among three siRNAs targeting SOCS1, SOCS1 mRNA and protein expression are most low in SOCS1-siRNA1 which is the best interference siRNA sequence. (A) Expression of SOCS1 mRNA in each cell line after transfection by Real Time-PCR; (B) Expression of SOCS1 protein in each cell line after transfection by Western blot. 


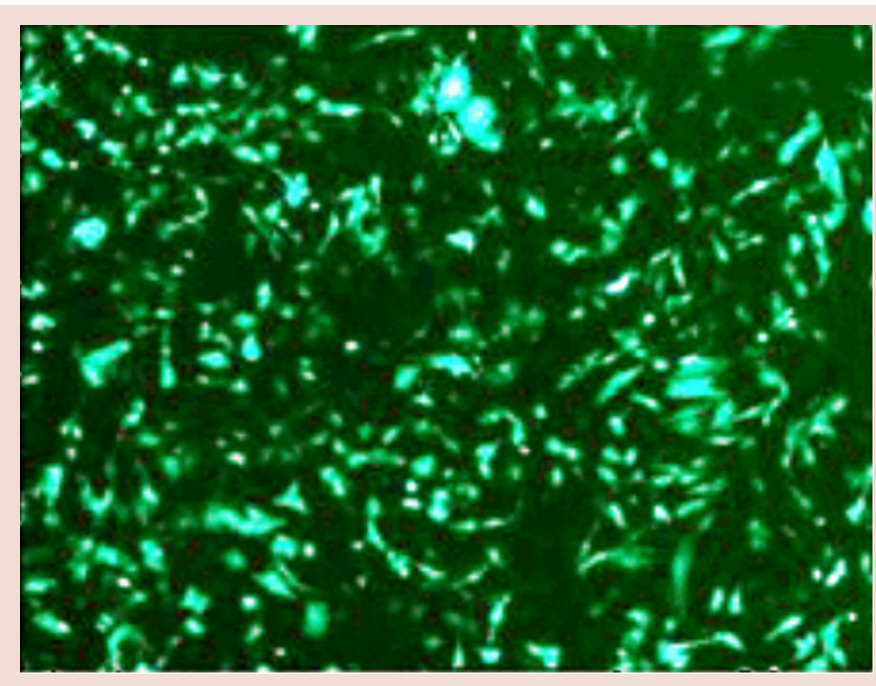

Figure 4: Fluorescence microscopy result of MCF7/ADM $96 \mathrm{~h}$ after lentiviral infection (MOI=6×120). The condition of lentiviral infected cells is good and the percent of cells expressing green fluorescent protein is more than $90 \%$.

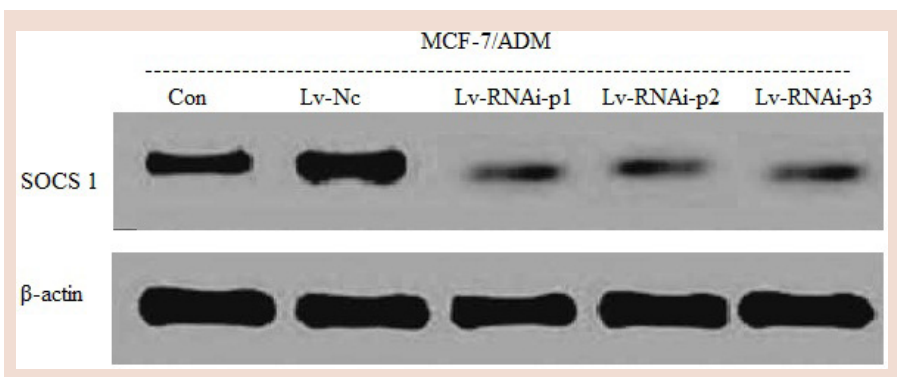

Figure 5: We infected lentiviral expressed vector into MCF7/ADM cells $(\mathrm{MOI}=6)$ and obtained high inhibition efficiency of protein expression $(>83 \%)$ while the expression lever of target gene had no change in various clone ages.

SOCS1 participates in the MDR process of malignant tumor cells, but to date there are no studies on the effects of SOCS1 gene in MDR of breast cancer, therefore our study mainly focuses on this question and aims to understand effects of SOCS1 gene in MDR of breast cancer as well as its mechanisms looking forward to bringing new hopes for reversing MDR by gene therapy.

First of all, we tested SOCS1 expression in breast cancer tissues and adjacent normal tissues from 41 patients by immunohistochemistry, and the results revealed a positive expression rate of SOCS1 (34.1\%), which was significantly lower than that of normal group. This result is similar to results from studies on renal cell carcinoma ${ }^{23}$ and lung cancer, ${ }^{24}$ indicating SOCS1 gene may be a tumor-suppressing gene and its downregulating expression may be correlated with breast cancer. In our study the expression rate of SOCS1 had no correlations with age, tumor size, lymph node metastasis, TNM stage, pathological type, ER and PR expression, which is consistent with the results of the study of Hao on gastric cancer. ${ }^{12}$ This indicates SOCS1 is not correlated with development and metastasis of breast cancer. Whether SOCS1 really participates in development and metastasis of breast cancer and its related mechanisms will be further studied. Owing to the fact P-gp plays a key role in MDR of breast cancer, ${ }^{25}$ we focused on studying the correlation between SOCS1 and P-gp, and the results revealed that P-gp expression in breast cancer was positively correlated with SOCS1 expression. Additionally we tested

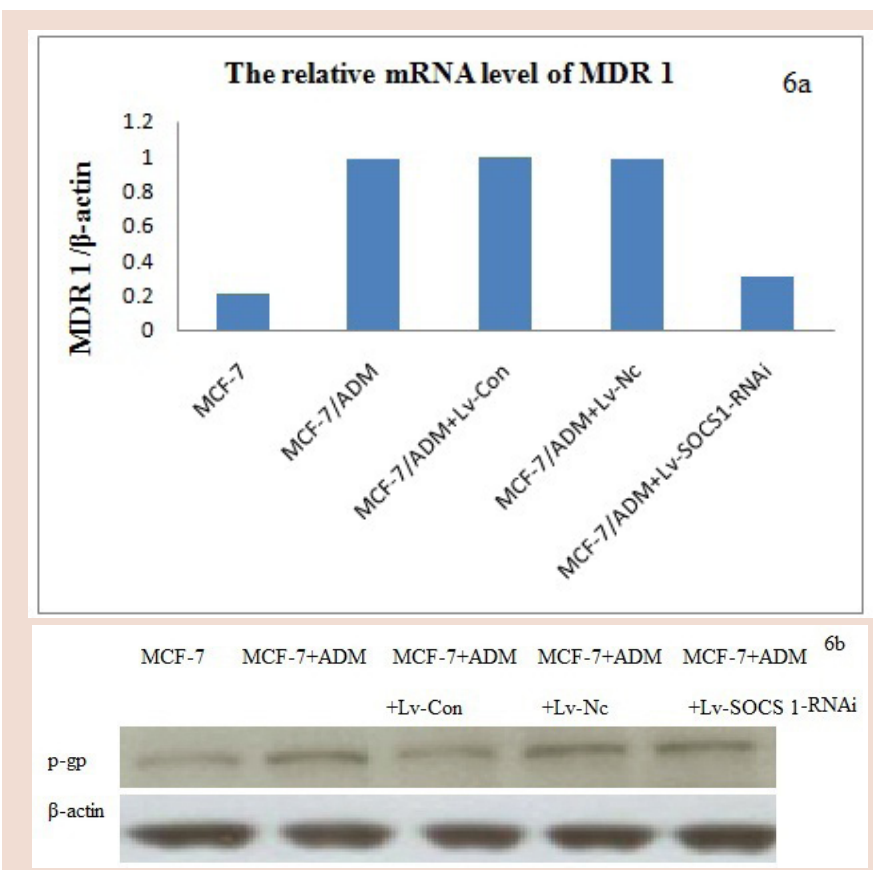

Figure $\mathbf{6} \mathbf{a}, \mathbf{b}$ : The expression of MDR1mRNA and P-gp in MCF7/ADM cell lines had a statistical significance before and after RNAi $(p \leq 0.01)$. These results indicated that SOCS1 may affect MDR-1 to inhibit P-gp expression in drug-resistant MCF7/ADM cell. (A) Expression of MDR1 mRNA in breast cancer cell line by Real Time-PCR; (B) Expression of MDR1 mRNA in breast cancer cell line by Western blot.

SOCS1 expression in many cell lines including MDA-MB-453, MDAMB-231, MCF7, MCF7/ADM, ZR-75-30, SK-BR-3 by Western blot assays, the results of which indicated that the MCF7/ADM cell line with MDR characteristics had the highest SOCS1 expression compared to the parental MCF7 line. These studies primarily revealed that SOCS1 participates in MDR of breast cancer, and may be correlated with the MDR1 gene, which lays the foundations for our follow-up experiments.

RNA interference is post-transcriptional gene silencing mediated by double-stranded RNA with a specific sequence. ${ }^{26}$ As a simple and effective technique taking the place of gene knockout, RNA interference is highly sequence-specific, highly effective, and fast-acting. Since 1998 when RNA interference was found, it had been widely used in studying gene functions and disease treatments. ${ }^{27}$ Compared to artificial siRNA, shRNA with a stem-loop structure is more efficient and specific. ${ }^{28}$ In our study we adopted lentiviral vector-mediated shRNA to inhibit expression of the SOCS1 gene. First of all, we used design software to analyze the SOCS1 full-length gene, and eventually three shRNA sequences were designed targeting its CDS region. Each sequence included two complementary single-stranded DNA templates, both of which were connected by 5'-CTTCCTGTCAGA-3', and followed by RNA PolyIII polymerase transcription termination sites (TTTTT) on the back, meanwhile BamHI and EcoR I restriction enzyme sites were added on both ends of the template strand separately. After this sequence was transferred into cells, RNA chain folded into shRNA with hairpin structure.

These shRNA may be cut into siRNA by intracellular Dicer enzyme. Compared to chemical synthesis and in vitro enzymatic synthesis, expression vector may consistently produce siRNA by transcription within cells. Therefore the time of action of siRNA may be prolonged, which leads to silencing of the target gene. Additionally it is more reliable to select a stable cell line by lentiviral transfection. Owing to the fact lentivirus have almost no immunogenicity, therefore gene intervention 

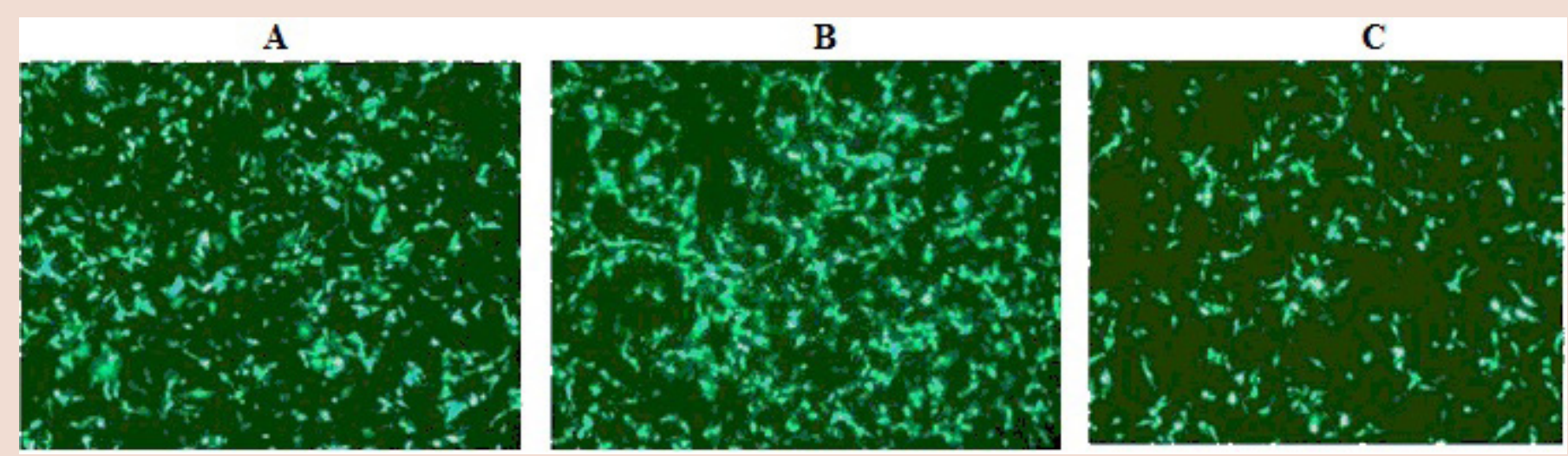

Figure 7: Fluogram of various breast cancer cell line stained by Rhodamine 123 (×400). (A): MCF7; (B): MCF7/ADM; (C): MCF7/ADM after RNAi.

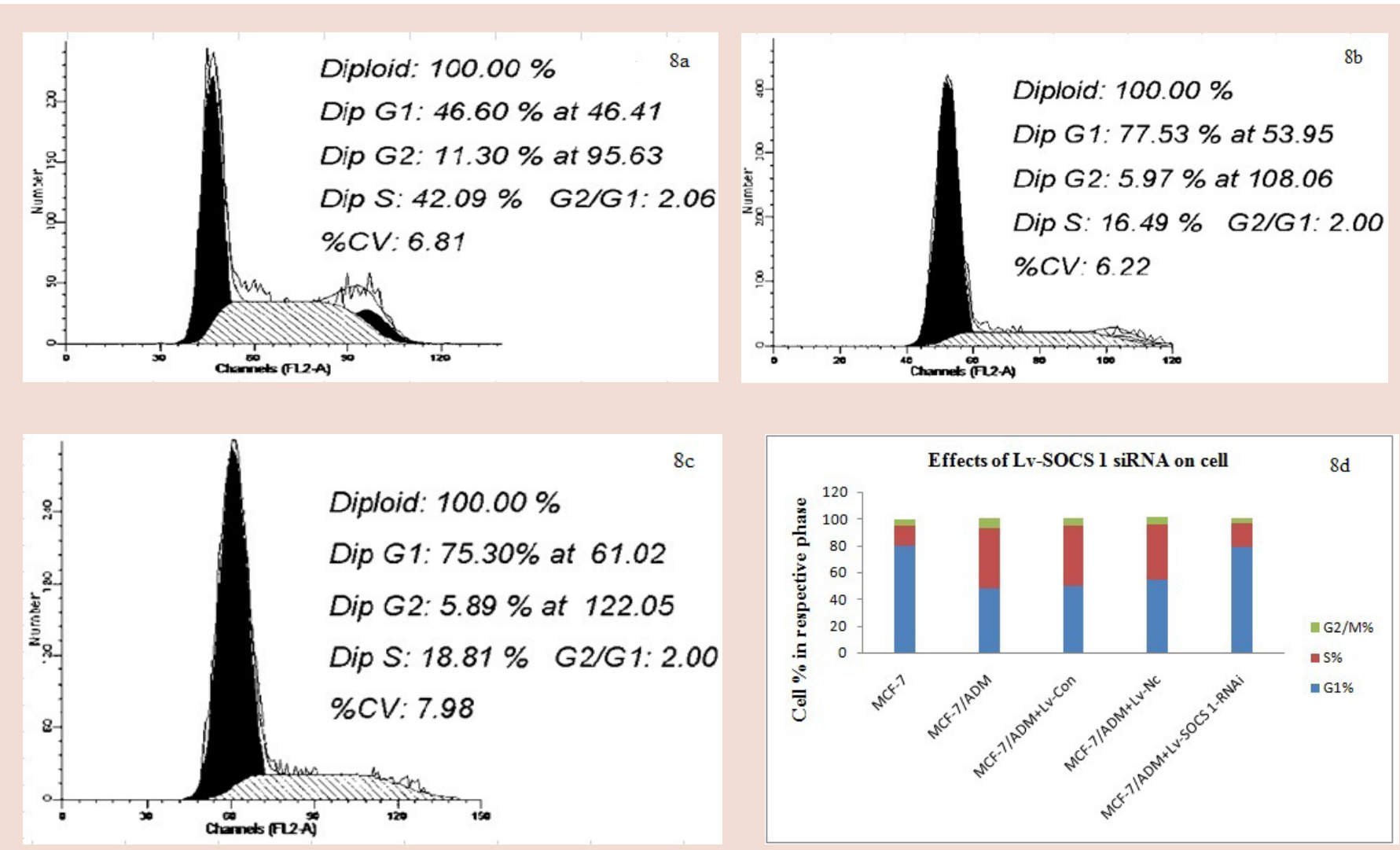

Figure 8a-d: Results from flow cytometry analysis showed that the cell cycle distribution was significantly affected by siRNA targeting SOCS1. (A): MCF7; (B): MCF7/ADM before RNAi; (C): MCF7/ADM after RNAi; (D): Proportion of cell cycle of various breast cancer cell lines.

by lentivirus should be one of the most effective methods. ${ }^{29}$ Compared to injection of siRNA produced by chemical synthesis or plasmid DNA, it has more advantages, which are beneficial for further in-vivo study. ${ }^{30,31}$ Our study adopted the third-generation lentiviral vector which had the least relativity with wild-type human HIV-1 virus, and this extremely increased the security of the vector. After we successfully transferred target gene into the purpose vector, lentivirus with no replicability was produced in 293TN cells to inhibit the SOCS1 gene. Results of Real-time PCR revealed that rate of SOCS1 gene silencing could reach up to $88 \%$. Western blot results indicated that the level of protein expression also achieved corresponding results (over 83\%). Additionally observation on different generations of cell cloning after infection by immunofluo- rescence found GFP expression did not change significantly with the increasing generation of cell clones. This demonstrated that expression of target gene in a stable cell line did not change with the increasing generation of cells. A series of tests revealed lentiviral transfection may effectively achieve RNA interference of the SOCS1 gene, and specifically inhibit SOCS1 gene expression in target cells.

After successfully performing RNA interference on the SOCS1 gene, we studied the changes of cell resistance of an MCF7/ADM cell line towards chemotherapy by IC50 value changes. Doxorubicin, paclitaxel, gemcitabine were selected as drugs commonly used in treating breast cancer, all of which have MDR characteristics and MDR1 gene plays a vital role in their resistances, therefore they are often used in studies 


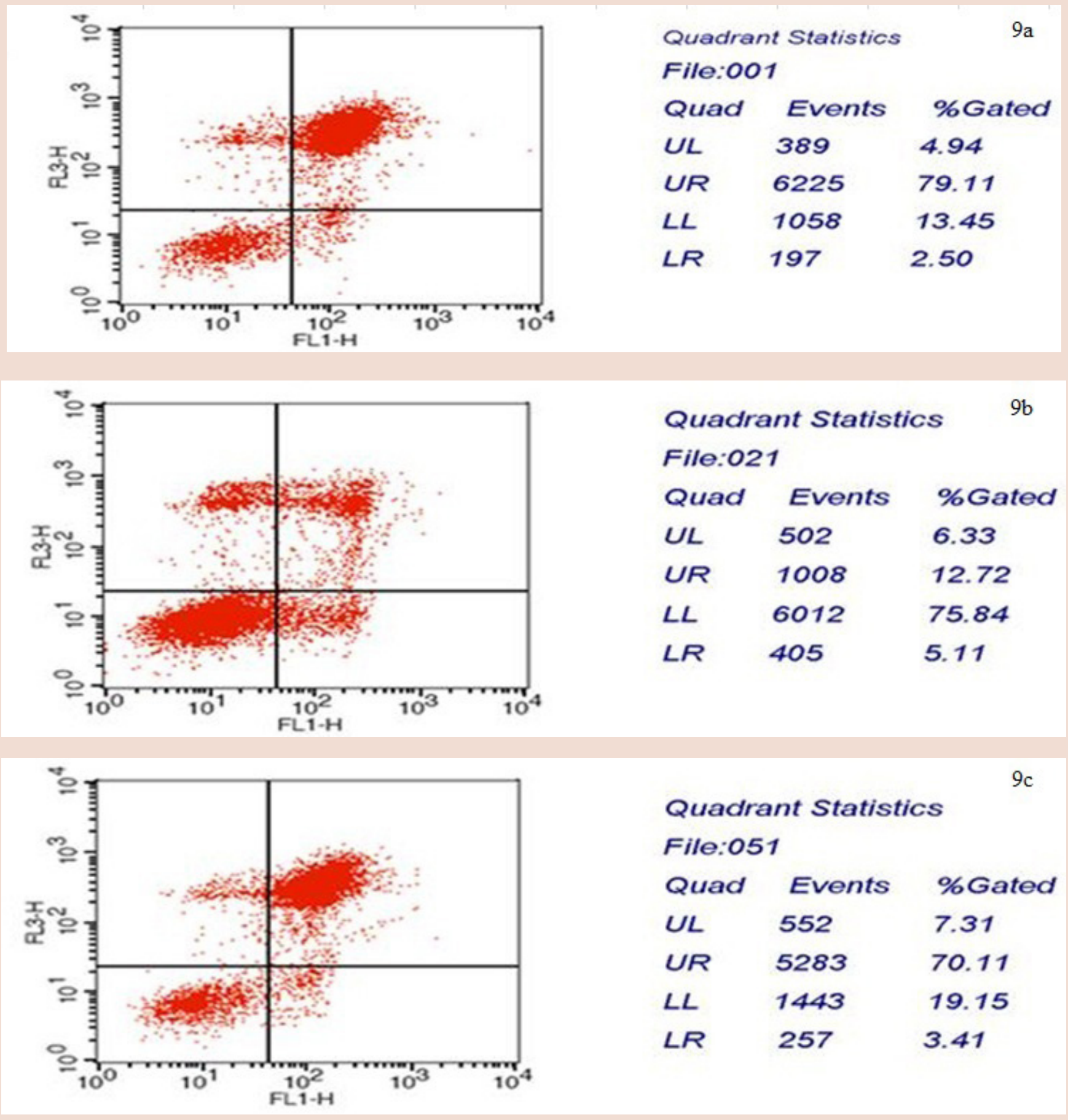

Figure 9a-c: Apoptosis of cells were determined by flow cytometry analysis. Compared with the cells without the treatment of SOCS1 siRNA, the apoptosis rate significantly increased after RNAi. (A): MCF7; (B): MCF7/ADM before RNAi; (C): MCF7/ADM after RNAi.

of MDR in breast cancer. ${ }^{32-34}$ The results revealed that when SOCS1 expression was suppressed, the resistance of cell lines towards the three medicines were all significantly decreased, which further confirmed that SOCS1 gene played a vital role in MDR of breast cancer, and we could overcome resistance of breast cancer by down-regulating expression of the SOCS1 gene. Therefore which mechanisms SOCS1 gene may adopt to regulate MDR of breast cancer is also a highlight in our study.

As mentioned above, the most important mechanism affecting MDR of breast cancer is drug resistance-associated proteins on the tumor cell membrane, especially P-gp encoded by MDR1. By the action of chemotherapy, expression of MDR1 on tumor cell membrane is up-regulated through many signal transduction pathways, and expression of P-gp which is encoded by MDR1 increases to protect tumor cells from apoptosis caused by chemotherapy. ${ }^{35}$ Inhibiting MDR1 gene expression for the prevention of and to overcome MDR of breast cancer may improve response of chemotherapy, and prognosis of patients. This study adopted Real-Time PCR and Western blot tests to examine the expression of MDR1 and P-gp, the results of which revealed that when the SOCS1 gene 


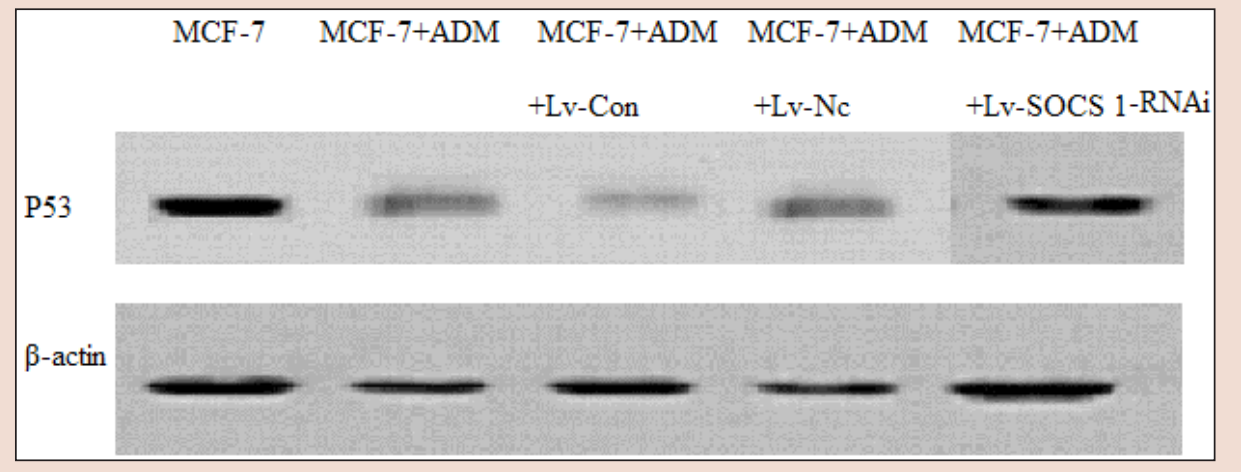

Figure 10: Expression of P53 Protein in Breast Cancer Cell Lines Before and After RNAi by Western blot.

in the MCF7/ADM cell line was down-regulated, expression of MDR1 mRNA and P-gp also decreased significantly. This indicated that SOCS1 gene may induce MDR of breast cancer by enhancing MDR1 gene. Additionally, P-gp may bind with rhodamine specifically, ${ }^{36}$ and can transfer rhodamine out of cells. This process is the same as P-gp transferring anti-tumor drugs. Rhodamine itself is fluorescent, which is easy to detect, therefore we often use Rhodamine efflux experiments to determine cells' ability to pump drugs, and P-gp on cell membranes can be detected. Staining results revealed that the fluorescence intensity of MCF7/ADM decreased significantly compared to its parental MCF7, indicating the efflux function of resistant cells was relatively strong, whereas RNA interference may induce silencing of the SOCS1 gene, and staining fluorescence intensity increased significantly, which demonstrated that the efflux ability of cells had decreased. All results above further revealed that SOCS1 gene may affect MDR of breast cancer by regulating expression of MDR1.

Abnormal proliferation is a basic characteristic of tumor cells, and the fundamental reason for uncontrolled proliferation lies in an imbalance of cell cycle regulation. Studies on the cell cycle of tumor cells have revealed that resistant cell lines have more S-phase cells, which promoted proliferation of tumor cells. Results from our study also found that MCF7/ADM cell line had more S-phase cells comparing to parental cells, but after RNA interference, G1-phase cells increased significantly with S-phase cells decreasing significantly $(p<0.001)$. This indicated that DNA synthesis was suppressed, and most cells were arrested in the G1 phase. Therefore we could confirm that SOCS1 may participate in MDR of breast cancer by regulating the cell cycle directly or indirectly. However there is a study finding that after SOCS1 gene expression was down-regulated by RNAi, S-phase cells increased significantly, which indicated that SOCS1 gene played the role of tumor suppressor gene and this was inconsistent with what we found. ${ }^{13}$ This discrepancy can be explained by heterogeneity of tumor cells or other reasons that need further study.

Apoptosis is regulated by apoptosis-related genes. Abnormal apoptosis not only plays a vital role in tumor development, but also participates in MDR of tumor cells and co-induces MDR through other pathways, which makes tumor cells resistant to apoptosis induced by chemotherapy. ${ }^{37-39}$ Our study revealed that by the action of ADM, antiapoptosis ability of MCF7/ADM cell line was pretty strong, however, after performing RNAi on SOCS1 gene, its anti-apoptosis ability was also identical with its parental cell line. The study found that Bcl-2 expression in some tumor cell lines was up-regulated with Bax and Fas expression down-regulating. However after SOCS1 gene was suppressed, Bcl-2 expression decreased with increasing Bax and Fas expression, therefore MDR of cells was arrested. Studies confirmed that apoptosis-related genes affected MDR of cells by regulating apoptosis. Whether SOCS1 affected MDR of cells through these genes or other apoptosis-related genes in breast cancer will be our next research focus.

P53 plays a vital role during tumor development, and many studies have revealed that it participates in MDR. When P53 gene mutation, deletion occurs (Mutant p53), its regulation on apoptosis also becomes abnormal. ${ }^{40-41}$ Apoptosis induced by chemotherapy is suppressed, which leads to significantly increasing resistance of tumor cells. Besides regulating apoptosis, $\mathrm{P} 53$ also participates in regulation of P-gp, and gene mutation may promote MDR of tumor cells through specific activation of MDR1/ P-gp promoter. In our study we found that when SOCS1 gene expression was down-regulated, expression of wild-type P53 was up-regulated, which indicated that SOCS1 gene may down-regulate expression of wild-type P53. Therefore we assumed that SOCS1 gene may up-regulate MDR1 expression by regulating wild-type P53 expression, which leads to breast cancer cell resistance. Meanwhile we must emphasize that in our study we selected MCF7/ADM and its parental MCF7 cell line as targets, and whether this conclusion still holds for other breast cancer cell lines still needs further study.

\section{Experimental}

\section{Tissue Samples, Cell Lines and Cell Culture}

Forty-five breast cancer and adjacent benign tissue samples were obtained from patients who underwent surgical treatment at the Affiliated Panda Cancer Hospital, Bhubaneswar, Odisha, India. All samples were obtained from patients who gave informed consent to use excess pathological specimens for research purposes only. All patients had undergone no chemoradiotherapy or endocrine therapy preoperation. The multidrug resistant human breast carcinoma cell line MCF 7/ADM and along with their parental sensitive cell lines MCF7 were obtained from MTCC, Pune, India and the CAS cell storeroom respectively. MCF7 and MCF7/ADM cells were cultured in DMEM (Invitrogen, USA) in a $5 \% \mathrm{CO} 2$ incubator at $37^{\circ} \mathrm{C}$, supplemented with $10 \%$ fetal bovine serum and antibiotics.

\section{Design and Synthesis of Recombined Plasmid of siRNA Targeting SOCS1}

The complementary DNA sequence of SOCS1 (NM_020313.2) was obtained from Genbank. The potential target sequences for RNA interference (RNAi) were scanned with the siRNA Target Finder and 
Design Tool available at the Ambion Web site (http://www.ambion. com/techlib/misc/siRNA_finder.html). The designed SOCS1 siRNA sequences are: 5'-GATCCGGAGCCAGTAGAGACAGC TCTTCCTGTCAGAAGCTGTCTCTACTGGCTCCTTTTTG-3'(SOCS1siRNA1), 5'-GATCCGTG GGTTCTTCTAGGCAGCCTTCCTGTCAGAGCTGCCTAGAAGAACCCACTTTTTG-3' (SOCS1 siRNA2) and 5'-GATCCGTCAGCTTGTGGAAACTGCCTTCCTGTCAGAGCAGTTTCCACAAGC TGACTTTTTG-3'(SOCS1siRNA3). The forward and reverse sequences were synthesized, annealed and coloned into the BamHI/ EcoR I of pSIH1-H1-copGFP vector (System Biosciences, San Francisco, CA, USA), yielding recombinant plasmids psiSOCS1-1, psiSOCS1-2 and psiSOCS1-3. The inserted sequences were confirmed by DNA sequencing. These recombinant plasmids and controls (empty plasmid and siRNA-Negative-plasmid) were transfected into 293 cell with Lipofectmaine $^{\mathrm{TM}} 2000$ (Invitrogen) following the manufacturer's instruction to determine the most efficient interfering sequence.

\section{Recombinant Lentivirus Generation and Lentivirus Infection}

Lentivirus Package plasmids mix (System Biosciences) and selected psiSOCS1-1 were cotransfected into $293 \mathrm{TN}$ cells to package and produce lentiviral vector and viral titer was determined according to the gradient dilution method. Lentivirus $\left(1 \times 10^{4} \mathrm{IFU} / \mu \mathrm{L}\right)$ packaging of green fluorescent protein (GFP) was infected into MCF7/ADM at various volume to determined the best MOI value at which concentrations no virus toxicity effect on cells was found. Then the experimental group (MCF7/ADM+SOCS1-siRNA1) and control group (MCF7/ADM+LvNegative) were added viruses at MOIs of 6 and incubated in normal condition. $96 \mathrm{~h}$ after infection we selected cells in two holes of which the GFP brightness is the strongest and proceeded intermediate clone culture in experimental group.

\section{Real Time-PCR Analysis}

TRIZOL reagent (Invitrogen) was used to extract total RNA according to the protocol of the manufacturer. Reverse transcription was done by reverse transcriptase to synthesis cDNA. The polymerase chain reaction (PCR) primers used were as follows: As for SOCS1, 5'-CCTGTACGCCA ACACAGTGC-3' (forward), 5'-ATACTCCTGCTTGCTGATCC-3' (reverse) and for $\beta$-actin, 5'-CCTTCGGCCTGGTGGATGTCTTT-3' (forward), 5'-CTCCCGCTGCAGCTCTTTCACTTC-3' (reverse), while for MDR1, 5'-CCGTGGGGCAAGTCAGTTCA (forward), CCGGTCGGGTGGGAT AGTTG-3' (reverse) and for $\beta$-actin, 5'-CCTGTACGCCAACACAGTGC-3' (forward), 5'-ATACTCC TGCTTGCTGATCC-3' (reverse). PCR products were separated on a $1 \%$ agarose gel, visualized and photographed under ultra violet light. In relative quantitation measurement the date was analyzed by CT value compare to $\beta$-actin mRNA.

\section{Western Blot Analysis}

For protein analysis, the cell lysates were harvested and detected by western blots. The antibodies used were monoclonal antibodies against SOCS1 and P-gp (Sigma Chemicals, Mumbai, India), anti-P53 and anti$\beta$-actin (Sigma Chemicals, India) antibodies, Anti-mouse HRP-linked Antibody (Sigma Chemicals, India) and Anti-rabbit HRP-linked Antibody (Cell Signaling Technology, Pune, India). Densitometry analysis of protein levels was performed by using Scion-Image 4.0.2 software (USA).

\section{Detect IC50 Values of Cells against Various Chemotherapeutics}

Each group of cells (MCF7; MCF7/ADM; MCF7/ADM+SOCS1-RNAi) in logarithmic phase was seeded in culture plates of 96 wells at $37^{\circ} \mathrm{C}, 5 \%$ $\mathrm{CO} 2$. Each well was added various chemotherapeutics (epirubicin, paclitaxel, gemcitabine) in different doses. OD values of the cells were tested by the method of MTT after $24 \mathrm{~h}$ incubation. Cell Growth inhibition ratio $=(1$-average OD value of experimental group/average OD value of control group) $\times 100 \%$. IC $_{50}$ value was calculated.

\section{Cell Cycle Analysis}

Standard fluorescence-activated cell sorter analysis was used to determine the distribution of cells in cell cycle. Briefly, cells in logarithmic phase were seeded in culture plates of 6 wells containing DMEM+10\% FBS at $37^{\circ} \mathrm{C}, 5 \% \mathrm{CO}_{2}$ with the final cell density of $1 \times 10^{5}$ per well. Adherent cells then were collected by trypsinization and fixed with $70 \%$ ethanol overnight at $4^{\circ} \mathrm{C}$ after $24 \mathrm{~h}$ culture. After washing with phosphate-buffered saline (PBS), the cells were added $100 \mu \mathrm{L}$ PBS containing $100 \mu \mathrm{g} / \mathrm{mL}$ RNase A (Roche Diagnostics, Mannheim, Germany) and $50 \mu \mathrm{g} / \mathrm{mL}$ propidium iodide (Sigma Chemicals, India) and incubated for $30 \mathrm{~min}$ at room temperature. The samples were analyzed using a FACS flow cytometer (Pune, India). The cell cycle distribution was established by plotting the intensity of the propidium iodide signal, which reflects the cellular DNA content. Findings from at least 20,000 cells were collected and analyzed with the Cell Quest software (Pune, India).

\section{Apoptosis Analysis}

Apoptotic MCF7/ADM cells were quantified by annexin V-FITC staining, using a kit purchased from Pune, India. In brief, cells in logarithmic phase were seeded in culture plates of 6 wells containing DMEM+10\% FBS for $24 \mathrm{~h}$ and DMEM without FBS for $8 \mathrm{~h}$. Then cells were maintained in medium containing doxorubicin $(5 \mu \mathrm{g} / \mu \mathrm{L})$ at $37^{\circ} \mathrm{C}, 5 \% \mathrm{CO} 2$ for $24 \mathrm{~h}$. Cells were washed twice with ice-cold PBS and then resuspended in 500 $\mu \mathrm{L}$ binding buffer at a concentration of $1 \times 10^{6}$ cells $/ \mathrm{mL} .5 \mu \mathrm{L}$ Annexin $\mathrm{V}$-FITC and $5 \mu \mathrm{L}$ propidium iodide were added to these cells in order. The fluorescence was immediately determined by flow cytometry. Excitation wave length is $488 \mathrm{~nm}$. Green fluorescence of Annexin V-FITC was detected by FITC channel 1 (FL1) while red fluorescence of PI (FL2) was detected by PI channel.

\section{Rhodamine 123 Staining Test}

We paved coverslipd treated with $37 \%$ sulphuric acid on the bottom of culture plates of 6 wells. Cell suspension $\left(5 \times 10^{4}\right.$ cells $\left./ \mathrm{mL}\right)$ was harvested after trypsinization and seeded in 6 wells and cultured for $24 \mathrm{~h}$. Then we taken out coverslip and dropped Rh123 buffer $(2 \mu \mathrm{M})$ on it. After 45 min incubation $4 \%$ paraform-aldehyde solution was then added. We observed the staining condition of cells using fluorescence inverted microscope.

\section{Statistical Analysis}

All experiments were performed at least three times. Statistical analysis was performed using Student's $t$-test by SPASS13.0 software and statistical significance was expressed as ${ }^{\star} \mathrm{p}<0.05$.

\section{CONCLUSION}

MDR of breast cancer is an extremely complicated and consistently changing process, which is regulated by a series of genes and factors. In our study we confirmed that SOCS1 gene participates in this process, which was accomplished by regulation of MDR1 and P53 as well as influencing cell cycle and apoptosis. Meanwhile we also confirmed that performing RNAi on SOCS1 gene by a viral transfection approach may effectively reverse MDR of breast cancer, which provides a new way for improving therapeutic effects. This represents a promising gene therapy.

\section{ACKNOWLEDGEMENT}

The research was supported by UGC, New Delhi and the authors acknowledge thanks to the HOD, UDPS for his kind support throughout the study. 


\section{CONFLICT OF INTEREST}

The authors declare that they have no conflict of interests.

\section{ABBREVIATION USED}

SOCS1:Suppressors of cytokine signalling; MDR: Multi Drug Resistant,; Pgp: P-glycoprotein; GFP: Green Fluorescent Protein; MCF 7: Michigan Cancer Foundation 7; PCR: Polymerase Chain Reaction; TNM: Tumor Node Metastasis; GFP: Green fluorescent protein, DMEM: Dulbecco's Modified Eagle's Medium; FACS: Fluorescence-activated cell sorting; FITC: Fluorescein isothiocyanate.

\section{REFERENCES}

1. Boetes C. Update on screening breast MRI in high-risk women. Magn Reson Imaging Clin N Am. 2010;18(2);241-7.

2. Xiang YB, Jin F, Gao YT. Cancer survival in Shanghai, China, 1992-1995. IARC Sci Publ. 2011;162:55-68.

3. Seruga B, Hertz PC, Le LW, Tannock IF. Global drug development in cancer: A cross-sectional study of clinical trial registries. Ann Oncol. 2010;21(4);895-900

4. Kuo MT. Roles of multidrug resistance genes in breast cancer chemoresistance. Adv Exp Med Biol. 2007;608:23-30.

5. Chung HC, Rha SY, Kim JH, Roh JK, Min JS, Lee JS et al. P-glycoprotein: the intermediate end point of drug response to induction chemo-therapy in locally advanced breast cancer. Breast Cancer Res. Treat. 1997;42(1):65-72.

6. Taheri M, Mahjoubi F, Omranipour R. Effect of MDR1 polymorphism on multidrug resistance expression in breast cancer patients. Genet Mol Res. 2010;9(1):34-40

7. Patwardhan G, Gupta V, Huang J, Gu X, Liu YY. Direct assessment of P-glycoprotein efflux to determine tumor response to chemotherapy. Biochem Pharmacol. 2010;80(1):72-9

8. DönmezY, Gündüz U. Reversal of multidrug resistance by small interfering RNA (siRNA) in doxorubicin-resistant MCF-7 breast cancer cells. Biomed Pharmacother. $2011 ; 65(2): 85-9$

9. Shibayama H, Takai E, Matsumura I, Kouno M, Morii E, Kitamura Y et al. Identification of a cytokine-induced antiapoptotic molecule anamorsin essential for definitive hematopoiesis. J Exp Med. 2004;199(4):581-92.

10. Hodge DR, Hurt EM, Farrar WL. The role of IL-6 and STAT3 in inflammation and cancer. Eur J Cancer. 2005;41(16):2502-12.

11. Amodio N, Bellizzi D, Leotta M, Raimondi L, Biamonte L, D'Aquila $P$, et al. miR-29b induces SOCS-1 expression by promoter demethylation and negatively regulates migration of multiple myeloma and endothelial cells. Cell Cycle. 2013; 12(23):3650-62

12. Flowers OL, Subramaniam PS, Johnson MH. A SOCS-1 peptide mimetic inhibits both constitutive and IL-6 induced activation of STAT3 in prostate cancer cells; Oncogene. 2005;24(12):2114-20

13. LiY, Liang C, Ma H, Zhao Q, LuY, Xiang Z, et al. miR-221/222 promotes S-phase entry and cellular migration in control of basal-like breast cancer. Molecules. 2014;19(6):7122-37.

14. Zhang J, Li H, Yu JP, Wang SE, Ren XB. Role of SOCS1 in tumor progression and therapeutic application.; Int J Cancer. 2012;130(9):1971-80.

15. Yoshikawa H, Matsubara K, Qian GS, Jackson P, Groopman JD, Manning JE, et al. SOCS-1, a negative regulator of the JAK/STAT pathway, is silenced by methylation in human hepatocellular carcinoma and shows growth-suppression activity.; Nature Genetics. 2001;28(1):29-35.

16. Galm O, Yoshikawa H, Esteller M, Osieka R, Herman JG. SOCS-1, a negative regulator of cytokine signaling, is frequently silenced by methylation in multiple myeloma; blood journal. 2003;101 (7):2784-8

17. Lage $\mathrm{H}$. An overview of cancer multidrug resistance: A still unsolved problem. Cell Mol Life Sci. 2008;65(20):3145-67.

18. Bae SK, Gwak J, Song IS, Park HS, Oh S. Induction of apoptosis in colon cancer cells by a novel topoisomerase I inhibitor Top In. Biochem Biophys Res Commun. 2011;409(1)75-81.
19. Bansal T, Akhtar N, Jaggi M, Khar RK, Talegaonkar S. Novel formulation approaches for optimising delivery of anticancer drugs based on P-glycoprotein modulation. Drug Discov Today. 2009;14(21)1067-74.

20. Walsh N, Larkin A, Kennedy S, Connolly L, Ballot J, Ooi W, et al. Expression of multidrug resistance markers ABCB1 (MDR-1/P-gp) and ABCC1 (MRP-1) in renal cell carcinoma. BMC Urol. 2009;9(1):6

21. Xing $H$, Wang $S$, Weng $D$, Chen G, Yang $X$, Zhou J, et al. Knock-down of P-glycoprotein reverses taxol resistance in ovarian cancer multicellular spheroids. Oncol Rep. 2007;17(1)117-22.

22. Osafo J, Wei Y, Kenth G, Goodyer CG. Growth Hormone during Development; Reviews in Endocrine and Metabolic Disorders. 2005;6(3):173-82.

23. Wu KL, Miao H, Khan S. JAK kinases promote invasiveness in VHL-mediated renal cell carcinoma by a suppressor of cytokine signaling-regulated, HIF-independent mechanism. Am J Physiol Renal Physiol. 2007;293(6):F1836-46.

24. Shimada K, Serada S, Fujimoto M, Nomura S, Nakatsuka R, Harada E, et al. Molecular mechanism underlying the anti-proliferative effect of suppressor of cytokine signaling-1 in non-small-cell lung cancer cells.; Cancer Sci. 2013; 104(11):1483-91.

25. Kuo MT. Roles of multidrug resistance genes in breast cancer chemoresistance. Adv Exp Med Biol. 2007;608:23-30.

26. Hannon GJ. RNA interference. Nature. 2002;418(6894):244-51.

27. Sumimoto $H$, Kawakami Y. The RNA silencing technology applied by lentiviral vectors in oncology. Methods Mol Biol. 2010;614:187-99.

28. Sliva K, Schnierle BS. Selective gene silencing by viral delivery of short hairpin RNA. Virol J. 2010:7(248):248.

29. Ni Y, Sun S, Oparaocha I, Humeau L, Davis B, Cohen R, et al. Generation of a packaging cell line for prolonged large-scale production of high-titer HIV-1-based lentiviral vector. J Gene Med. 2005;7(6):818-34.

30. Raoul C, Abbas-Terki T, Bensadoun JC, Guillot S, Haase G, Szulc J. et al. Lentiviral-mediated silencing of SOD1 through RNA interference retards disease onset and progression in a mouse model of ALS. Nat Med. 2005;11(4):423-8.

31. Liu J, Guan X, Ma X. Regulation of IL-27 p28 gene expression in macrophages through MyD88-and interferon-gamma-mediated pathways. J Exp Med. 2007;204(1):141-52.

32. Liang $Y$, Gao H, Lin SY, Goss JA, Brunicardi FC, Li K siRNA-based targeting of cyclin $E$ overexpression inhibits breast cancer cell growth and suppresses tumor development in breast cancer mouse model. PLoS One. 2010;5(9):e12860.

33. Niu G, Castro CH, Nguyen N, Sullivan SM, Hughes JA. In vitro cytotoxic activity of cationic paclitaxel nanoparticles on MDR-3T3 cells. J Drug Target. 2010;18(6):468-76.

34. Bergman AM, Adema AD, Balzarini J, Bruheim S, Fichtner I, Noordhuis P et al. Antiproliferative activity, mechanism of action and oral antitumor activity of CP-4126, a fatty acid derivative of gemcitabine, in in vitro and in vivo tumor models. Invest New Drugs. 2011;29(3):456-66.

35. Mechetner E, Kyshtoobayeva A, Zonis S, Kim H, Stroup R, Garcia R, et al. Levels of multidrug resistance (MDR1) P-glycoprotein expression by human breast cancer correlate with in vitro resistance to taxol and doxorubicin. Clin Cancer Res. 1998;4(2):389-98.

36. Molnár J, Kars MD, Gündüz U, Engi $H$, Schumacher U, Van Damme EJ, et al. Interaction of tomato lectin with $A B C$ transporter in cancer cells: Glycosylation confers functional conformation of P-gp. Acta Histochem. 2009;111(4):329-33

37. Ahsan H, Reagan-Shaw S, Breur J, Ahmad N. Sanguinarine induces apoptosis of human pancreatic carcinoma AsPC-1 and BxPC-3 cells via modulations in Bcl-2 family proteins. Cancer Lett. 2007;249(2):198-208.

38. Holcomb B, Yip-Schneider M, Schmidt CM. The role of nuclear factor kappaB in pancreatic cancer and the clinical applications of targeted therapy. Pancreas 2008;36(3):225-35.

39. Liu F, Xie ZH, Cai GP, Jiang YY. The effect of survivin on multidrug resistance mediated by P-glycoprotein in MCF-7 and its adriamycin resistant cells. Biol. Pharm Bull. 2007;30(12):2279-83.

40. Schwartz D, Rotter V. p53-dependent cell cycle control: Response to genotoxic stress. Semin Cancer Biol. 1998;8(5):325-36

41. Oue T, Yoneda A, Uehara S, Yamanaka H, Fukuzawa M. Increased expression of multidrug resistance-associated genes after chemotherapy in pediatric solid malignancies. J Pediatr Surg. 2009;44(2):377-80.

\section{SUMMARY}

- SOCS1 have wide and imperative capacities, particularly because of its close correlation with malignant tumors

- SOCS1 inhibits the expression of the target gene.

- SOCS1 reduces drug resistance and the expression of MDR1 mRNA and P-gp in MCF7/ADM cell lines.

- SOCS1 take part in breast cancer MDR by managing MDR1 and P53 expression, changing cell cycle and enhancing the anti-apoptotic ability of cells. 


\section{PICTORIAL ABSTRACT}

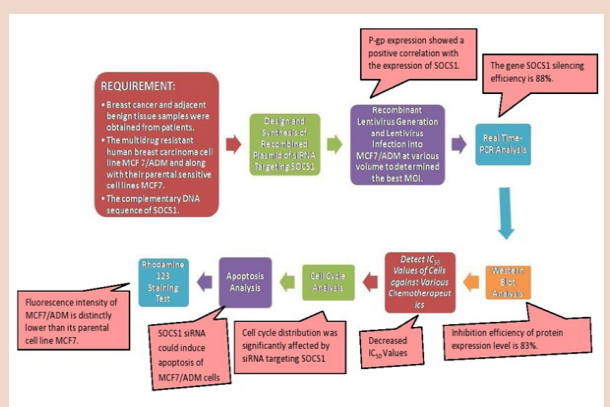

ABOUT AUTHORS

Dr. Debasish Pradhan: Is Specialist in Immunity and Breast Cancer Medicine. He is currently Sr Faculty at Dept. of Pharmaceutical Sciences, Utkal University. He is a recognized expert in nutritional medicine, immunity and cancer. Six (06) no of PhD degree have been awarded under his Supervision. He has written over 50 articles on natural medicines and one book "Fundamentals and principles in Pharmacology". He has all the degrees and publication in the research of EthnoMedicine/Herbal Medicine/Natural Medicine and a total experience of thirteen years.

Dr. Gitanjali Tripathy: Is continuing as a Lecturer in Govt Polytechnic College at Bhubaneswar. She has contributed 21 no of Publication and A Text Book in Pharmacology with good referred journals. She did his Doctoral programme in Pharmacy from Dept of Pharmaceutical Sciences, Utkal University, Bhubaneswar, Odisha. 